\title{
Der Städtebummler
}

SEBASTIAN BANERT Hohe Anforderungen sowie Zeit- und Leistungsdruck führen immer häufiger zu psychischen Erkrankungen und damit zu Arbeitsausfällen. In seiner Bachelorarbeit untersuchte Sebastian Banert, wie man eine zeitgemäße ergotherapeutische Intervention - die sich von der klassischen Arbeitstherapie abgrenzt bei der Behandlung von Arbeitsstörungen effektiv gestalten kann.

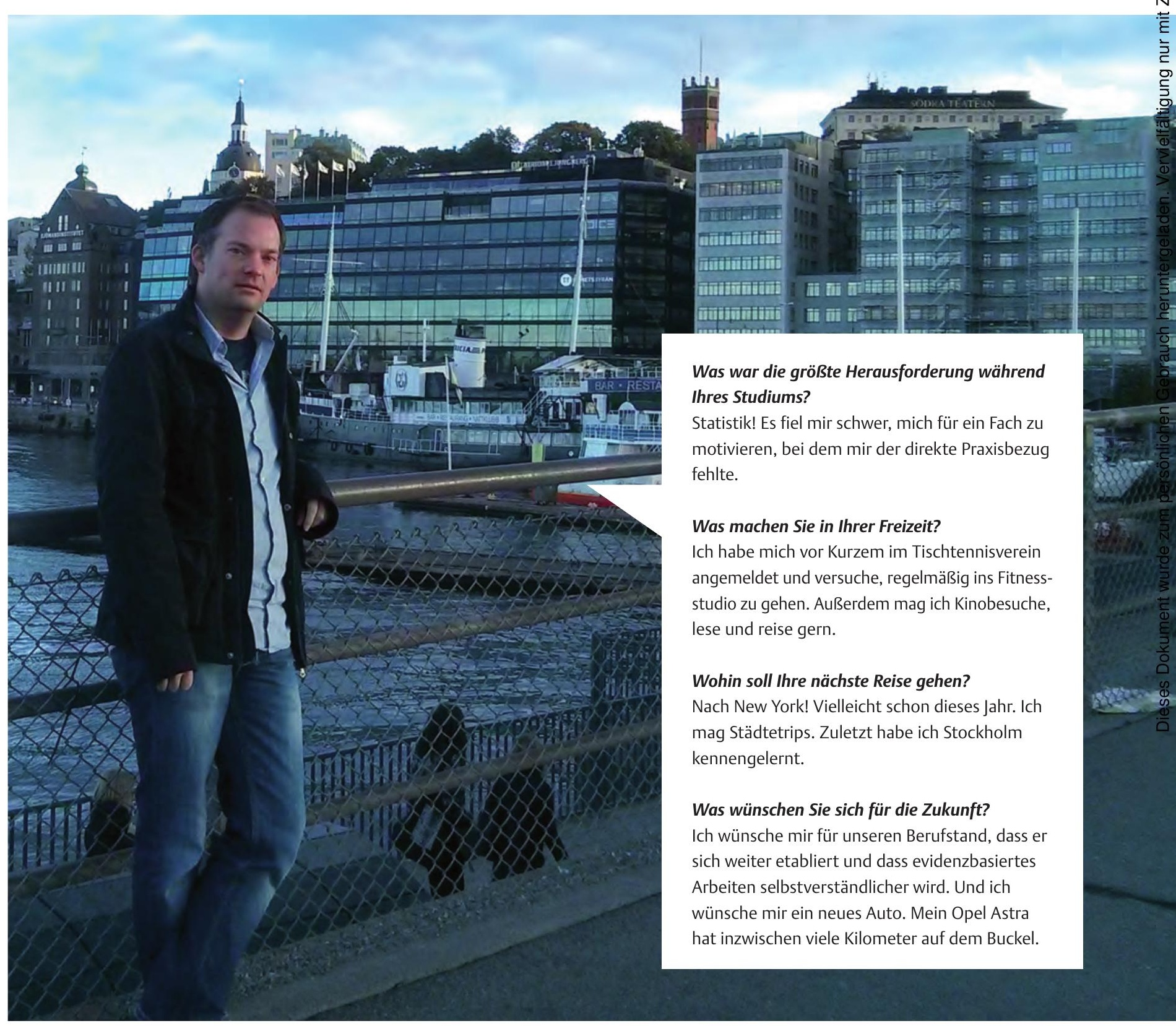




\section{Weiterentwicklung des Ergotherapie- Angebotes bei Arbeitsstörungen}

\section{Sebastian Banert ...}

... ist 33 Jahre alt und Ergotherapeut in der LWL Klinik Warstein - Fachklinik für Psychiatrie, Psychotherapie und Psychosomatik. Im Kompetenzzentrum für Integration unterstützt er Menschen mit Arbeitsstörungen.

Die Ergotherapieausbildung absolvierte der gebürtige Lippstädter 2005 in seiner Heimatstadt. Dort war er auch bis 2010 für die LWL Kliniken tätig. Anschließend wechselte er nach Warstein und nahm das Studium zum Bachelor of Arts für Medizinalfachberufe an der Diploma Hochschule University of Applied Sciences im Studienzentrum Bochum auf. Im April 2013 schloss er sein Studium mit dem Schwerpunkt "Lehre“ erfolgreich ab.

Der Ergotherapeut hat Lust auf mehr. Er kann sich beispielsweise vorstellen, in Zukunft Fortbildungen zu geben und ein Masterstudium aufzunehmen.

\section{Die Bachelorarbeit}

Leistungsanforderungen und Arbeitsdruck nehmen zu und damit auch die Zahl der Menschen, die dem nicht dauerhaft gewachsen sind. Sebastian Banert hat im Kompetenzzentrum für Integration der LWL Klinik täglich mit Klienten mit Arbeitsstörungen zu tun. So fand er auch zum Thema seiner Bachelorarbeit. Er beschreibt darin die Veränderungen am Arbeitsmarkt und die damit verbundenen negativen Auswirkungen. Mittels Literaturrecherche stellte er sich der zentralen Frage, wie man eine zeitgemäße ergotherapeutische Intervention - die sich von der klassischen Arbeitstherapie abgrenzt - bei der Behandlung von Arbeitsstörungen effektiv gestalten kann.

\section{Ergebnisse}

Sebastian Banert beschreibt, dass Arbeitnehmer vermehrt Belastungen wie langen Anfahrtswegen, Leiharbeit und flexibleren Arbeitszeitmodellen ausgesetzt sind. Daraus können Arbeitsstörungen wie Burn-out, Mobbing, Prokrastination (chronisches Aufschieben) und Gratifikationskrisen (Leistung wird zu wenig honoriert) resultieren. Die Folgen können ernst zu nehmende somatische und psychische Erkrankungen sein. Die Arbeitstherapie, die in verschiedenen Einrichtungen angewendet wird, beinhaltet das Trainieren von Grundarbeitsfähigkeiten in Arbeitsateliers, also in künstlich angelegten Situationen. Die Übertragung in den realen Arbeitsalltag fällt den Klienten oftmals schwer, denn die Transferleistung ist hoch. Diesem alten Konzept steht das Supported Employment (Unterstützte Beschäftigung) gegenüber. Hierbei trainiert der Klient an einem realen Arbeitsplatz und wird von seinem Job Coach begleitet und gefördert. Der Coaching-Ansatz regt den Klienten zur Selbstbefähigung an und gleicht einem Problemlösungsprozess, der das individuelle Trainieren und Reflektieren realer Ziele und Situationen impliziert. Auf diese Weise fällt ihm der Transfer in den Berufsalltag leichter.

Ergotherapeuten reflektieren zusammen mit den Klienten Fortschritte sowie Probleme und können dementsprechend die Therapie anpassen. Die Evidenz des Supported Employment ist bereits wissenschaftlich belegt. Es eröffnet Ergotherapeuten in ihrer Rolle als Berater einen handlungsorientierten, realitätsnahen Therapieansatz, welcher Partizipation und Inklusion ermöglicht.

\section{Fazit}

Zusammenfassend hält Sebastian Banert fest, dass ...

$>$ Ergotherapeuten über Wissen und Möglichkeiten verfügen, um Arbeitsstörungen wie Burn-out, Mobbing und Prokrastination zu behandeln.

$>$ neben dem Ziel der beruflichen Rehabilitation die Selbstbefähigung des Klienten und das Entwickeln von Bewältigungsstrategien im Zentrum der ergotherapeutischen Intervention stehen sollten. Der Klient kann so belastende Situationen frühzeitig erkennen, seine Ressourcen nutzen und einer Eskalation des Problems aktiv entgegenwirken.

$>$ die Berufstätigkeit dazu beiträgt, die Persönlichkeit und die soziale Einbindung zu stabilisieren sowie einen positiven Einfluss auf die Gesundheit zu nehmen. In der Rehabilitation sollte man eine zügige Rückkehr in die Arbeitswelt anstreben, um Hospitalisierungsprozesse und gesellschaftliche Ausgrenzung zu umgehen.

Nora Sieweke

$\rightarrow$ Banert S. Weiterentwicklung psychiatrischer ergotherapeutischer Angebote bei Arbeitsstörungen im Kontext neuer beruflicher Anforderungen. Bachelorarbeit an der Diploma Hochschule University of Applied Sciences; 2012 